\title{
AGN Spectra as Seen by the Infrared Space Observatory: First Results
}

\author{
D. Lutz, R. Genzel, and E. Sturm
}

Max-Planck-Institut für Extraterrestrische Physik, Postfach 1603, 85740 Garching, Germany

A. F. M. Moorwood

European Southern Observatory, Garching, Germany

E. Oliva and A. Marconi

Osservatorio Astrofisico di Arcetri, Firenze, Italy

H. Netzer

School of Physics and Astronomy, Tel Aviv University, Tel Aviv, Israel

\begin{abstract}
We discuss $2.5-45 \mu \mathrm{m}$ spectra of the Circinus galaxy and of Cen A, obtained with the Short Wavelength Spectrometer (SWS) on board the Infrared Space Observatory. The large number of detected ionic fine structure lines, observable also in visually obscured sources, provides strong constraints on the shape of the ionizing spectrum, which is found to exhibit a UV bump peaking at $\sim 70 \mathrm{eV}$ in the case of Circinus. Pure rotational emission of molecular hydrogen, directly probing warm molecular gas, can for the first time be detected in external galaxies.
\end{abstract}

\section{Introduction}

The advent of the Infrared Space Observatory (ISO) has opened the possibility of sensitive mid-infrared spectroscopy of active galactic nuclei. The $2.5-45 \mu \mathrm{m}$ range is particularly interesting for AGN research since it contains fine-structure transitions of many ionization stages of abundant elements. They provide excellent diagnostic tools for ionized media on account of the wide range of excitation potentials covered (up to $300 \mathrm{eV}$ ) and the inherently low sensitivity of the line fluxes and density-sensitive ratios to changes in electron temperature. The low extinction at mid-infrared wavelengths allows direct observation of the narrow-line region of visually obscured AGNs. Pure rotational lines of molecular hydrogen probe the warm molecular phase, and [FeII] transitions trace partially ionized zones. 


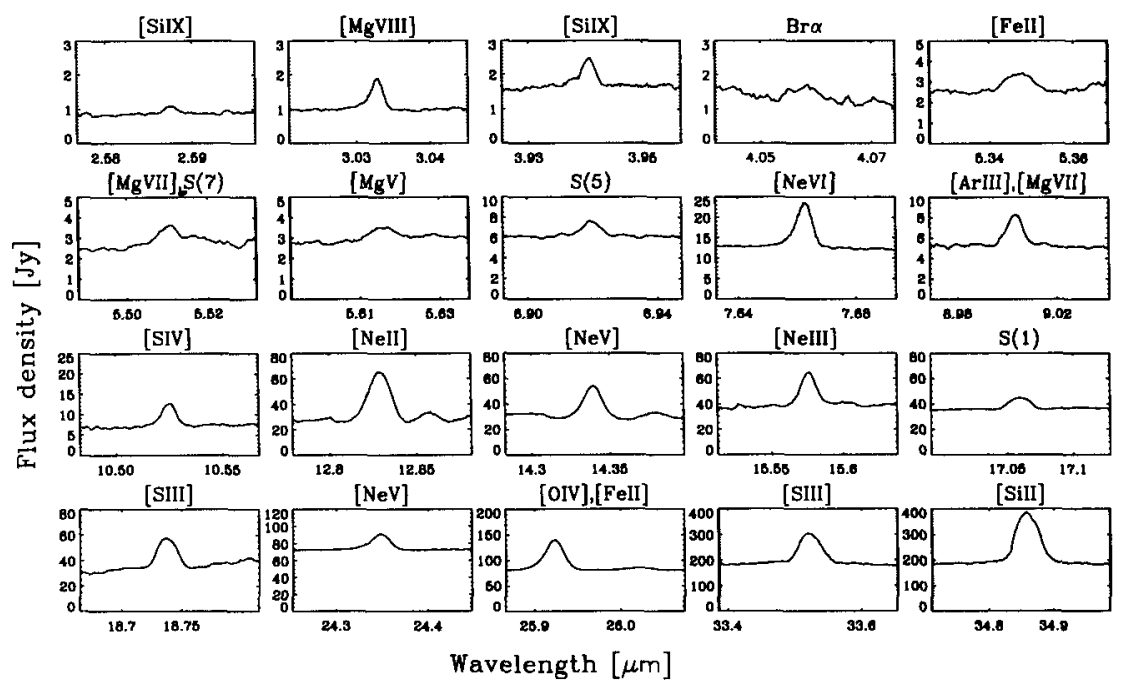

Figure 1. Individual line scans of Circinus. Some structure in the spectra between 12 and $28 \mu \mathrm{m}$ is due to residual instrumental fringing.

\section{Observations}

The Circinus galaxy was observed as a Seyfert 2 template due to its proximity $(4 \mathrm{Mpc})$, brightness, and narrow high-excitation lines. We obtained both a 2.5$45 \mu \mathrm{m}$ full spectrum aiming at a complete census of spectral features (Sturm et al. 1997, this volume) and individual line scans targeting at detection of faint lines and determination of line profiles (Fig. 1). For the visually obscured narrow-line region of Cen A, similar individual line scans have been obtained, with observations to date not yet including the highest-excitation lines.

\section{Emission-Line Spectrum and Nuclear Continuum}

Ionic emission lines detected in Circinus include [Fe II], [Ne II], and [S III], which are most likely dominated by the Circinus starburst ring that is partly included in the SWS aperture, intermediate-excitation lines like [S IV] and [Ne III], which may originate both in the starburst and the AGN, and high-excitation lines of [O IV], [Nev], [Ne VI], [MgV], [MgVII], [MgVIII], and [Si IX] which arise in gas photoionized by the AGN. We have used the [Nev] $14.3 \mu \mathrm{m} / 24.3 \mu \mathrm{m}$ flux ratio to measure a density of $5000 \mathrm{~cm}^{-3}$ for the highly ionized gas of Circinus.

The line widths inferred (taking into account the instrumental profile) are in the range $150-300 \mathrm{~km} \mathrm{~s}^{-1}$. The high-excitation lines are narrow for an AGN. The low-excitation lines are found to be wider, probably due to their larger spatial extent which leads to a wider profile due to both instrumental effects and rotational velocities. The prominent blue wing on the highest-excitation [Si IX] line (note the low extinction, $A_{3.94 \mu \mathrm{m}} \approx 0.05 A_{V}$ ) is attributed to the fact that this coronal line originates in outflowing gas within the ionization cone. Less 


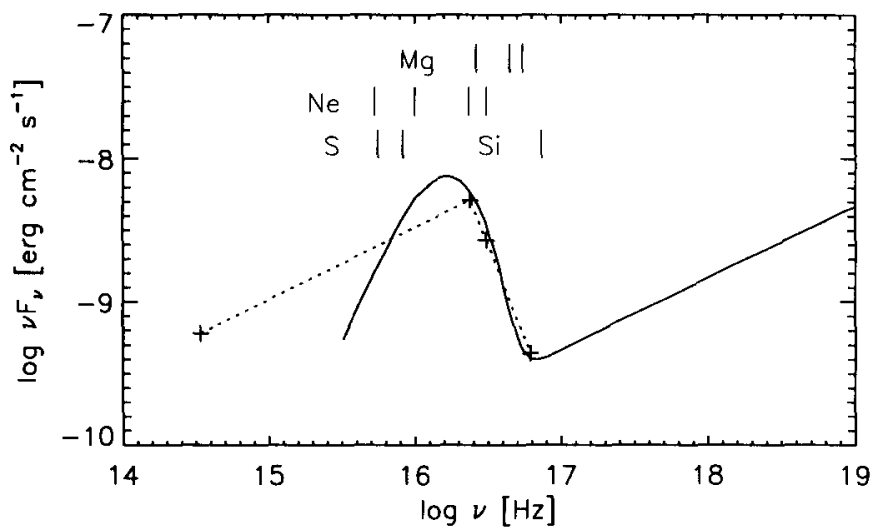

Figure 2. Inferred ionizing spectrum of Circinus. The frequencies needed to create some of the species detected by $I S O$ are indicated.

pronounced blue wings are also visible in the profiles of other high-excitation lines.

We have used the photoionization codes CLOUDY and ION to investigate the spectral energy distribution of the obscured central ionizing source (Moorwood et al. 1996). Using the line fluxes measured with $I S O$ and additional constraints, e.g., from visible/near-infrared coronal-line observations (Oliva et al. 1994) and $A S C A$ X-ray observations (Matt et al. 1996), we infer an ionizing continuum shape as shown in Fig. 2. The main result is the need to invoke a UV bump peaking at about $70 \mathrm{eV}$ in the $\nu \mathrm{F}_{\nu}$ representation of Fig. 2. The shape of the high-energy side of this UV bump is well constrained by the ISO lines and can be equally well fit by a broken power law (dashed line) and by a blackbody-like bump superposed on the high-energy $\mathrm{F}_{\nu} \propto \nu^{-0.5}$ continuum. The low-energy side is less well determined due to the interference of starburst emission in the low-energy lines. The models reproduces most of the observed $I S O$ and visible/NIR fluxes in the $50-320 \mathrm{eV}$ ionization energy range to within a factor of 2 , which is very satisfactory in view of remaining uncertainties in observations and atomic data. One interesting exception is [O IV] $26 \mu \mathrm{m}$ which is found to be $\sim 2.5$ times brighter than modeled. Observations of the narrow-line region of Cen $\mathrm{A}$ have not yet reached the level of detail of the Circinus data. Line fluxes from Neon ions up to [Ne vi], however, clearly indicate lower excitation than in Circinus.

The good quantitative agreement between observations of high-excitation lines and the Circinus AGN photoionization model adds confidence to the use of lines like [Nev] $14.3,24.3 \mu \mathrm{m}$ and [O IV] $26 \mu \mathrm{m}$ as AGN indicators for highly obscured sources of uncertain nature, such as ultraluminous IRAS galaxies. 


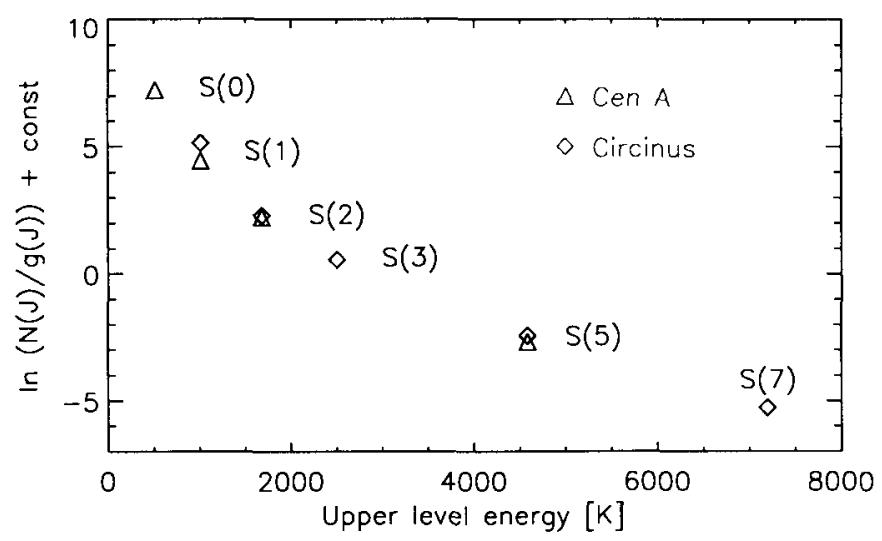

Figure 3. Upper level populations, divided by statistical weight, for the rotational transitions of $\mathrm{H}_{2}$ measured in Circinus and Cen A.

\section{Molecular Hydrogen Emission}

Strong rotational lines of molecular hydrogen are detected in both Circinus and Cen A. A plot of derived level population, divided by statistical weight, versus energy of the upper level (Fig. 3) demonstrates that the fluxes are inconsistent with thermal equilibrium at a single temperature, which would correspond to symbols following a straight line. The lower transitions $S(0), S(1), S(2)$ most likely are of thermal origin in gas at an average temperature of about $200 \mathrm{~K}$, as expected, for example, in photodissociation regions. The higher transitions like $S(5)$ or $S(7)$ arise in warmer and dense gas (e.g., shocks) or may have a fluorescent origin. The ability to detect modest quantities of warmer gas - the observed S(5) fluxes in Circinus and Cen A, for example, could be explained by about $5-10 \times 10^{3} M_{\odot}$ of molecular hydrogen at a temperature of $1000 \mathrm{~K}$ - and to penetrate absorbing column densities of the order $10^{23} \mathrm{~cm}^{-2}$ makes these transitions also a potential tool to detect X-ray illuminated gas close to an AGN. It should, however, be stressed that for sources with circumnuclear star formation like Circinus and Cen A, the observed emission is most likely dominated by that component. In fact, very similar level population diagrams as in Fig. 3 have been obtained for pure starbursts that ISO has observed to date.

\section{References}

Matt, G., et al. 1996, MNRAS, 281, L69.

Moorwood, A.F.M., Lutz, D., Oliva, E., Marconi, A., Netzer, H., Genzel, R., Sturm, E., \& de Graauw, Th. 1996, A\&A, submitted.

Oliva, E., Salvati, M., Moorwood, A.F. M., \& Marconi, A. 1994, A\&.A, 288, 457.

Sturm, E., Lutz, D., \& Genzel,R., 1997, this volume. 